\title{
Application of Solar-Electric Propulsion to Robotic Missions in Near-Earth Space
}

\author{
Gordon R. Woodcock* and John Dankanich ${ }^{\dagger}$ \\ Gray Research, Inc., 655 Discovery Drive, Suite 300, Huntsville Alabama $35806^{*}$
}

\begin{abstract}
Interest in applications of solar electric propulsion (SEP) is increasing. Application of SEP technology is favored when: (1) the mission is compatible with low-thrust propulsion, (2) the mission needs high total delta V such that chemical propulsion is disadvantaged; and (3) performance enhancement is needed. If all such opportunities for future missions are considered, many uses of SEP are likely. Representative missions are surveyed and several SEP applications selected for analysis, including orbit raising, lunar science and robotic exploration, and planetary science. These missions span SEP power range from $10 \mathrm{kWe}$ to about $100 \mathrm{kWe}$. A SEP design compatible with small inexpensive launch vehicles, and capable of lunar science missions, is presented. Modes of use and benefits are described, and potential SEP evolution is discussed.
\end{abstract}

\section{NOMENCLATURE AND ACRONYMS}

\begin{tabular}{|c|c|}
\hline$a$ & $=$ semi-major axis (sma) \\
\hline$e$ & $=$ eccentricity \\
\hline$F$ & Thrust component \\
\hline$k, k_{1}, k_{2}$ & undetermined constants \\
\hline$T_{1}$ & $=1+e \cos \theta$ \\
\hline$T_{2}$ & $=\operatorname{sqrt}\left(1+2 e \cos \theta+e^{2}\right)$ \\
\hline$T_{3}$ & $=1-e^{2}$ \\
\hline$T_{4}$ & $=e+\cos \theta$ \\
\hline$\alpha$ & $=$ pitch angle; unknown exponent \\
\hline$\beta$ & $=$ yaw angle \\
\hline$\gamma$ & $=$ path angle re local horizontal \\
\hline$\Delta V$ & $=\operatorname{delta} \mathrm{V}$ \\
\hline$\theta$ & $=$ true anomaly (from periapsis) \\
\hline$\lambda_{a}$ & $=$ LaGrange multiplier for sma \\
\hline$\lambda_{p}$ & $=$ LaGrange multiplier for plane \\
\hline$\mu$ & $=$ Earth geopotential \\
\hline$\omega$ & $=$ argument of periapsis \\
\hline
\end{tabular}

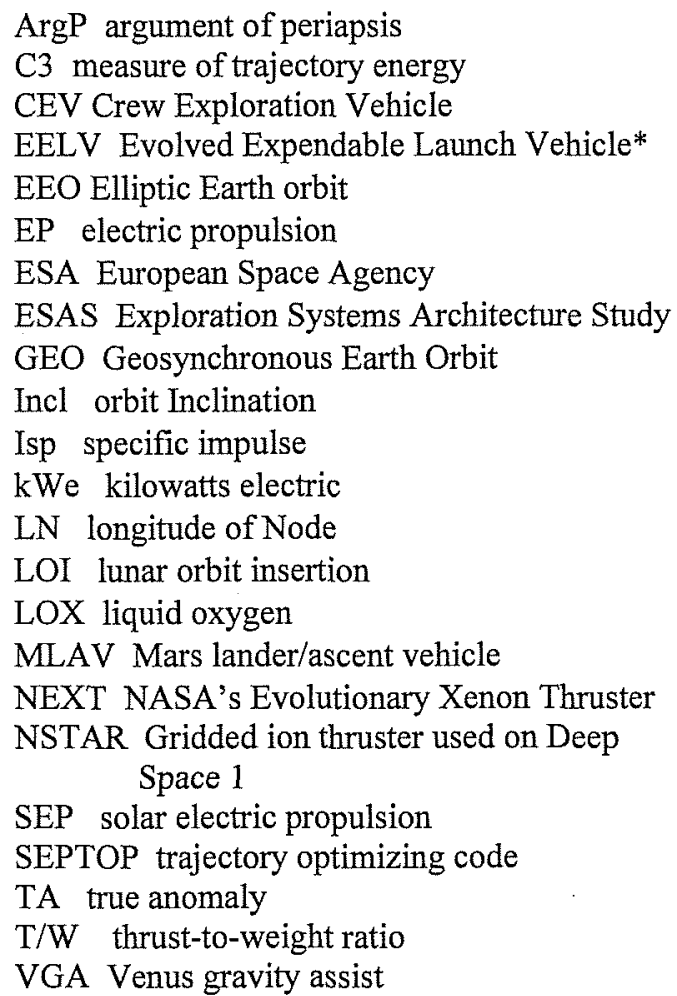

\section{INTRODUCTION}

Portions of this paper were originally presented at the Joint Propulsion Conference ${ }^{1} 2006$. The paper was requested for this meeting by the session chair. New material was added on a small SEP low-cost lunar mission. Solar-electric propulsion (SEP) has been widely applied to commercial communications satellites for stationkeeping in geosynchronous orbit and has recently seen use on scientific missions including NASA's Deep Space 1 and

\footnotetext{
*Senior Engineer, Gray Research Inc., 655 Discovery Drive Suite 300, Huntsville Alabama 35806; Associate Fellow

${ }^{\dagger}$ Aerospace Engineer, Gray Research Inc., 655 Discovery Drive Suite 300, Huntsville Alabama 35806
} 
ESA's SMART-1 missions. Solar-electric propulsion (SEP) is becoming of interest for application to a wide range of missions. The benefits of SEP are strongly influenced by system element performance, especially that of the power system. Solar array performance is increasing rapidly and promises to continue to do so for another 10 to 20 years (Fig. 1). At the same time, cost per watt is decreasing. Radiation hardness is increasing. New concepts for how to design a SEP are emerging. These improvements lead to changes in the best ways to apply SEP technology to missions, and broadening of the practical uses of SEP technology compared to competing technologies.

It is timely to discuss some of the emerging uses of SEP, and reasonable steps to advancing the technology to make it a sound choice for these applications.

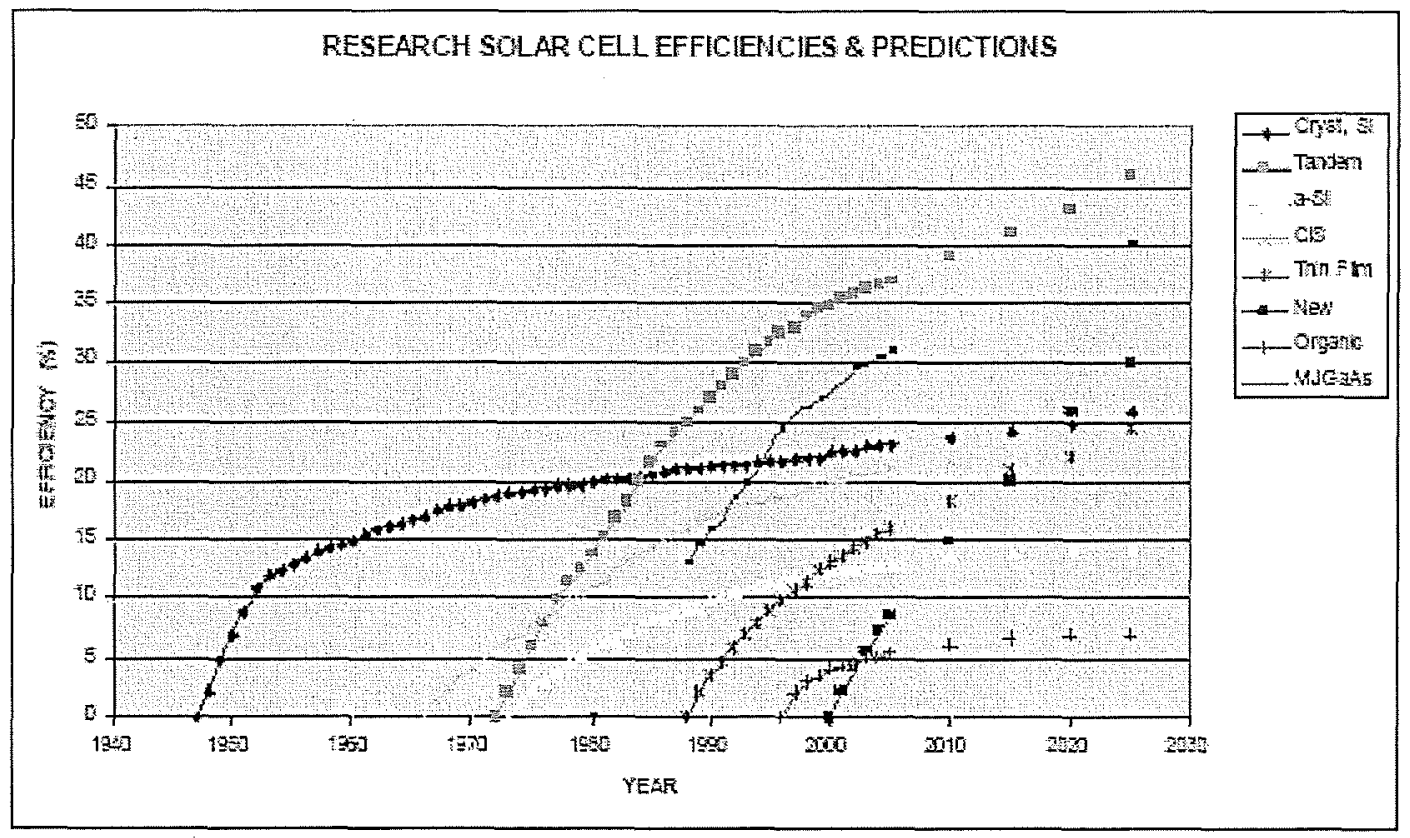

Figure 1. Historical and Projected Solar Cell Performance ${ }^{1}$

\section{MISSION APPLICATIONS}

Application of SEP technology is generally favored when: (1) the mission is compatible with low-thrust propulsion, i.e., no need to produce accelerations comparable to planetary gravity fields and adequate time to achieve the mission ideal delta $\mathrm{V}$; (2) the mission needs high total delta $\mathrm{V}$ such that chemical propulsion is disadvantaged; and (3) performance enhancement is needed to make the mission compatible with existing launch capabilities, or to provide cost reduction.

Table 1 presents a list of missions by category with potential SEP applications. The categories are borrowed from a survey of mission needs for advanced in-space propulsion, conducted by NASA's In-Space Propulsion Technology organization in 2004. Missions discussed in this paper are underlined.

\section{ORBIT RAISING}

Orbit raising may fit the criteria set forth above, depending on trip time considerations. In the near future we may expect to see communications satellites delivered to geosynchronous orbit (GEO) using some of their stationkeeping SEP to assist the orbit raising process by completing the GEO insertion.

More ambitious use of SEP is possible, but requires higher electric propulsion power than installed on current comsat designs. For example, a 2-ton satellite with $25 \mathrm{kWe}$ installed power and $5 \mathrm{kWe}$ electric propulsion thruster power would need about $150-180$ days to do GEO insertion from a geosynchronous transfer orbit, depending on thrusting strategy. If the installed thrust were designed to utilize $80 \%$ of the available electric power $(20 \mathrm{kWe})$ the insertion time would be reduced by a factor of 4 , to $40-45$ days.

A dedicated SEP vehicle could operate at still higher power and return to low Earth orbit for re-use. The payload fraction (net useful GEO payload/total mass delivered to LEO) could be roughly doubled. This involves 25 to 50 $\mathrm{kWe}$ SEP power for typical current comsats, and improvements in SEP radiation hardness so that at least four total uses (round trips) can be obtained. SEP costs are not well established but an estimator of $\$ 2000 /$ Watt electric is a reasonable working number. Then a $50 \mathrm{kWe}$ SEP will cost $\$ 100$ million plus its launch costs. To recoup such an 
investment through reduction in subsequent comsat launch costs, it must be spread over at least three LEO-GEO round trips, preferably four or more. Also required is a reliable, simple, economic scheme for handing off a launched payload in LEO to the returning SEP vehicle. A suitable technology remains to be demonstrated, but it would appear to be not more difficult than the automated rendezvous and docking used by Russian space station missions for many years.

Issues of radiation and debris exposure, plane change and suitable starting orbits are discussed in the next few paragraphs.

Table 1. SEP Applications by Mission Regime

\begin{tabular}{|l|l|}
\hline \multicolumn{1}{|c|}{ Mission Regime } & \multicolumn{1}{|c|}{ Representative SEP Applications } \\
\hline $\begin{array}{l}\text { Regime 1 - Human Earth Orbit, near-Earth missions } \\
\text { (includes ISS) }\end{array}$ & Orbit adjust and makeup, including application to ISS \\
\hline $\begin{array}{l}\text { Regime 2 - Robotic LEO to near planets; Earth \& } \\
\text { space observation, planetary science and sample } \\
\text { return }\end{array}$ & $\begin{array}{l}\text { Robotic LEO to higher Earth orbits and the Moon; Mars } \\
\text { sample return, missions to NEOs, Venus }\end{array}$ \\
\hline $\begin{array}{l}\text { Regime 3 - Human HEO to Lunar; space transfer } \\
\text { humans/cargo with landing and bases }\end{array}$ & $\begin{array}{l}\text { Cargo mission support for human lunar and other Earth } \\
\text { vicinity missions. }\end{array}$ \\
\hline $\begin{array}{l}\text { Regime 4 - Robotic near Sun; includes Mercury and } \\
\text { solar probes/polar missions }\end{array}$ & $\begin{array}{l}\text { Near-sun or out of ecliptic missions requiring high delta } \\
\text { V; trade vs solar sails }\end{array}$ \\
\hline $\begin{array}{l}\text { Regime 5 - Human inner planets; Mars exploration \& } \\
\text { landing, asteroids, space transfer }\end{array}$ & $\begin{array}{l}\text { Humans and cargo to Mars and NEO and main belt } \\
\text { asteroids. }\end{array}$ \\
\hline $\begin{array}{l}\text { Regime 6 - Robotic outer planets; orbiters, probes, } \\
\text { landers and sample return missions }\end{array}$ & $\begin{array}{l}\text { All of these except landers and ascent vehicle } \\
\text { themselves. }\end{array}$ \\
\hline $\begin{array}{l}\text { Regime 7 - Human outer planets; Jupiter and Saturn } \\
\text { and their moons, landing and return }\end{array}$ & $\begin{array}{l}\text { Perhaps some portions of trajectories but these missions } \\
\text { need thrust far from Sun. }\end{array}$ \\
\hline $\begin{array}{l}\text { Regime 8 - Robotic beyond planetary system; Kuiper } \\
\text { Belt, Oort Cloud, interstellar missions }\end{array}$ & $\begin{array}{l}\text { Very high performance SEP possibly can inject these by } \\
\text { producing enough delta V near Sun. }\end{array}$ \\
\hline
\end{tabular}

\section{A. Starting Orbit}

SEP ascent from a low circular orbit experiences lengthy periods in the van Allen belts and significant periods in the LEO debris environment. Conventional launch vehicles pay a high performance price for higher circular orbits, including decreased payload and a restart of the upper stage. The best solution to this problem is an elliptic starting orbit. Launch vehicle performance to an elliptic starting orbit with low perigee is much better than to higher circular orbits, and does not require an upper stage restart.

Elliptic starting orbits pay off in reducing radiation and debris exposure time, as shown in Fig. 2. Also shown is a typical launch vehicle performance comparison with calculated and quoted ${ }^{2}$ data. These are approximate overall effects of elliptic starting orbits. At the price of reduced launch capability, major reductions in total time as well as time in hazardous environments can be obtained. The circular orbit yaw steering formula discussed below was used to generate these general trends. An elliptic orbit formula is also described below and is better for performance but
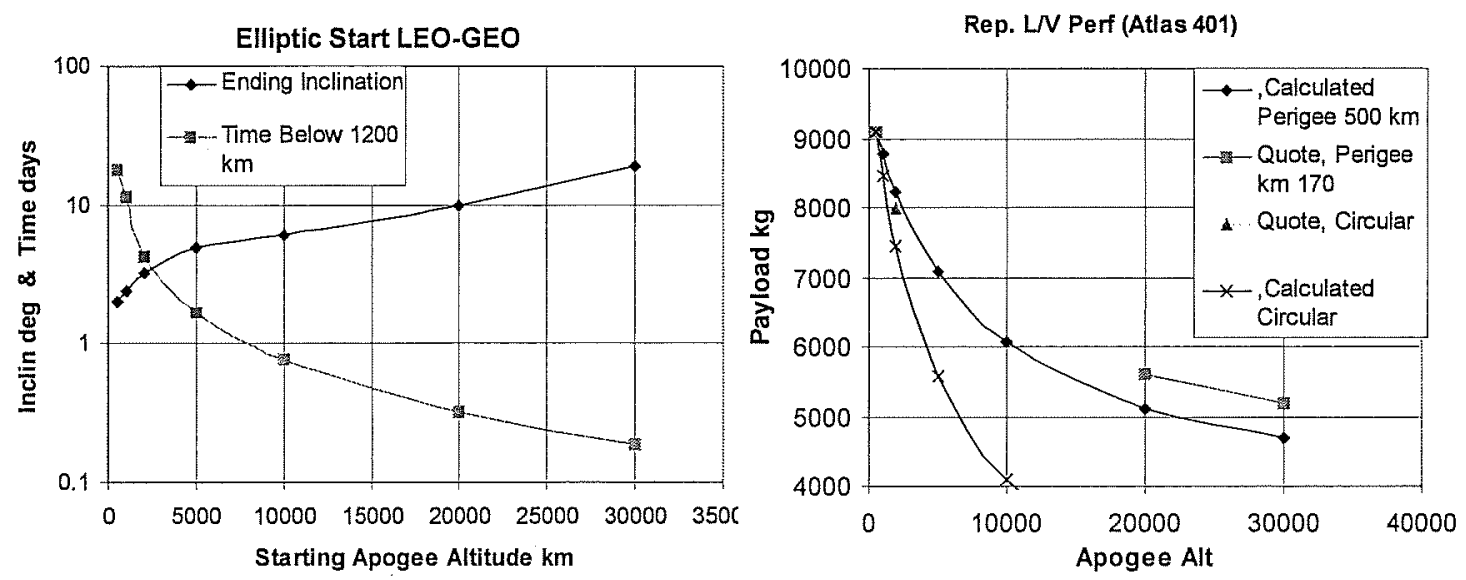

Figure 2. Trends for Elliptic Starting Orbits, as a Function of Starting Apogee 
these trends will be vary similar. The SEP can return to the starting orbit in about a month. For all-chemical propulsion the GEO/LEO payload ratio is about $20 \%$; even with an elliptic starting orbit, SEP is expected to double that. A point calculation using Atlas 401 performance found that a $35 \mathrm{kWe}$ SEP starting from a $400 \mathrm{x} 10,000$ orbit could deliver $3600 \mathrm{~kg}$ to GEO in about 130 days, compared to about half that for all-chemical propulsion.

\section{B. Thrusting Strategy}

Two questions are posed for orbit transfers: (1) should we thrust all the time or part of the time, and (2) should we thrust in the direction of flight or in some other direction?

The answer to the first question depends on whether we wish to minimize transfer time, or are we willing to trade transfer time for delta $V$ ? Numerical experiments were tried with a perigee hold approach (an in-plane pitch program is used to maintain perigee altitude while raising apogee). The pitch equation for perigee hold is

$$
\tan \alpha=\frac{T_{3}^{2} \sin \theta /\left(T_{1} T_{2}\right)}{2\left[(1+e) T_{3} T_{4} / T_{2}\right]}
$$

Perigee hold cost more delta $\mathrm{V}$ and more time to reach the desired orbit. There is no tradeoff of transfer time versus delta V. The next experiment tried was an apogee "gap", a period of non-thrusting centered on apogee. The idea is that thrusting at apogee contributes least to orbit raising. In this experiment, thrust was pointed in the direction of flight. A tradeoff did exist, as shown in Fig. 3. About 15 days of added trip time reduced delta $V$ by a few hundred $\mathrm{m} / \mathrm{s}$ and could increase payload several percent. Cases may exist where this tradeoff would allow use of a smaller launch vehicle, or enable a marginal payload to be delivered with adequate margin.

The next experiment involved maximizing the increase in apogee. One can write a differential equation for $\mathrm{dRa} / \mathrm{dp}$ where $\mathrm{Ra}$ is apogee radius and $\mathrm{p}$ is pitch angle, and set it equal zero to obtain the maximum equation,

$$
\tan \alpha=\frac{2(1-e) T_{2}-T_{3} T_{4} / T_{2}}{T_{3}^{2} \sin \theta /\left(T_{1} T_{2}\right)}
$$
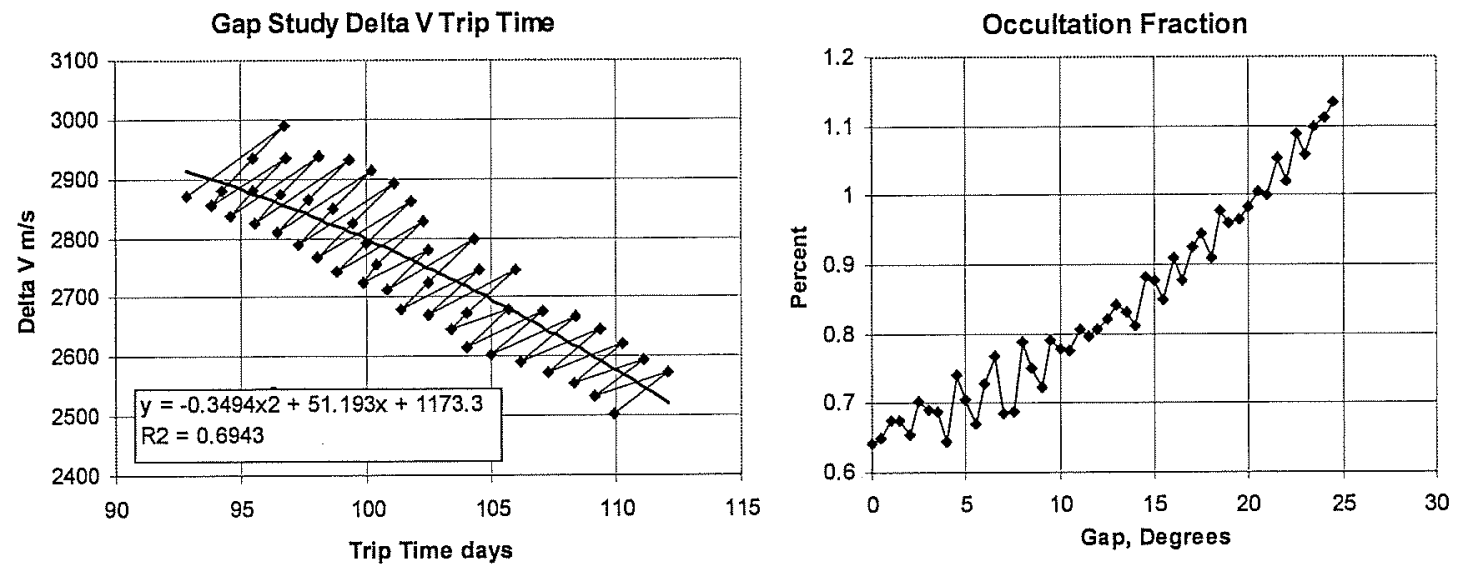

Figure 3: Delta V vs Trip Time and Occultation Effects for Apogee Gap Thrusting

The effects of this scheme are shown in Fig. 4. Over a small increase in semi-major axis, apogee increases faster than in the case of thrusting in the flight direction $(\alpha=0)$. However, the expression for $\partial \mathrm{r}_{\mathrm{a}} / \partial \mathrm{m}$ includes sma (semimajor axis) as a multiplier. After a while, slower growth in sma negates the advantage and it becomes a loss because smaller sma decreases $\partial \mathrm{r}_{\mathrm{a}} / \partial \mathrm{m}$. Further, slower growth of perigee leads to more occultation, which also leads to longer trip time. Therefore, this is not a good strategy.

The conclusion is that thrusting all the time in the flight direction provides minimum transfer time, but shutting off thrust over an arc centered on apogee offers a tradeoff of more payload for longer trip time. However, this "apogee gap" slows increase in perigee, therefore increasing radiation and debris exposure.

\section{Plane Change During Orbit Raising}

Orbit raising frequently requires plane change, as in ascent from LEO at 28.5 degrees inclination to GEO at zero inclination. The delta $\mathrm{V}$ added for plane change is significant and should be minimized. This can be done using a 
general-purpose low-thrust trajectory optimization routine ${ }^{3}$, but the approach used for this paper was to derive simple GN\&C laws for this specific application.
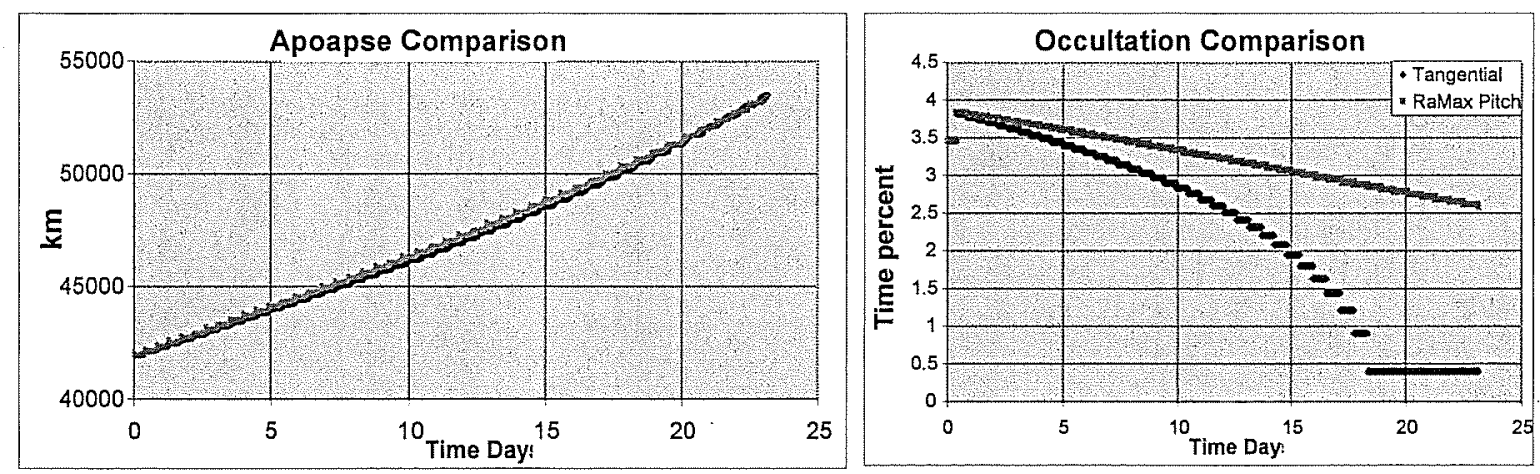

Figure 4: Results of Apogee Maximization Simulation

Using a low-thrust approximation (the orbit remains nearly circular) we can operate on orbit parameters to find the optimum transfer, noting that instantaneous orbit altitude increase is

$\mathrm{da} / \mathrm{d} \Delta \mathrm{V}=2 \mathrm{a}^{3 / 2} / \mathrm{sqr} t(\mu) \cos \beta$, and instantaneous plane change is

$\mathrm{dP} / \mathrm{d} \Delta \mathrm{V}=\operatorname{sqrt}(\mathrm{a} / \mu) \cos \theta \sin \beta$, where $\beta$ is out-of-plane yaw angle.

Writing a Hamiltonian, $H=\lambda_{a}\left(2 a^{3 / 2}\right) \cos \beta / \operatorname{sqrt}(\mu)+\lambda_{p} a^{1 / 2} \cos \theta \sin \beta / \operatorname{sqrt}(\mu)-d \Delta V$.

We find $\tan \beta=\lambda_{\mathrm{p}} \cos \theta /\left(2 \lambda_{\mathrm{a}} \mathrm{a}\right)$. This tells us that yaw steering oscillates back and forth along the orbit path, with magnitude of the yaw to be determined, and expected to be a function of $\lambda_{\mathrm{p}}, \lambda_{\mathrm{a}}$, and $\mathrm{a}$. $\lambda_{\mathrm{p}}$ turns out to be constant, and $\lambda_{a}$ is not analytically integrable. It could be integrated numerically, but from the form of the differential equation, a solution was presumed to look like $\lambda_{\mathrm{a}}=\mathrm{k}_{1} \exp \left(\mathrm{k}_{2} \mathrm{a}^{\alpha}\right)$. One might also experiment with a form $\lambda_{\mathrm{a}}=\mathrm{k}_{1} \mathrm{a}^{\alpha 1} \exp \left(\mathrm{k}_{2} \mathrm{a}^{\alpha 2}\right)$ but so far we have not done so. Further, a known very good guidance law is $1 /\left(\lambda_{\mathrm{a}} \mathrm{a}\right)=$ $\mathrm{ka}^{\alpha}$ (power law), so we compared new guidance laws with that.

As shown in Fig 5, the exponential power law is better than the simple power law but by only $2 \mathrm{~m} / \mathrm{s}$. The best exponential multiplier was about 0.75 and the best exponent 0.6 . The best exponent for the power law is about 0.85 . The two laws are compared at the lower left; they are almost the same. The rate of plane change with delta $\mathrm{V}$ increases at higher altitude, as one would expect; velocity is less, so less delta $V$ is needed to obtain a given amount of plane change.

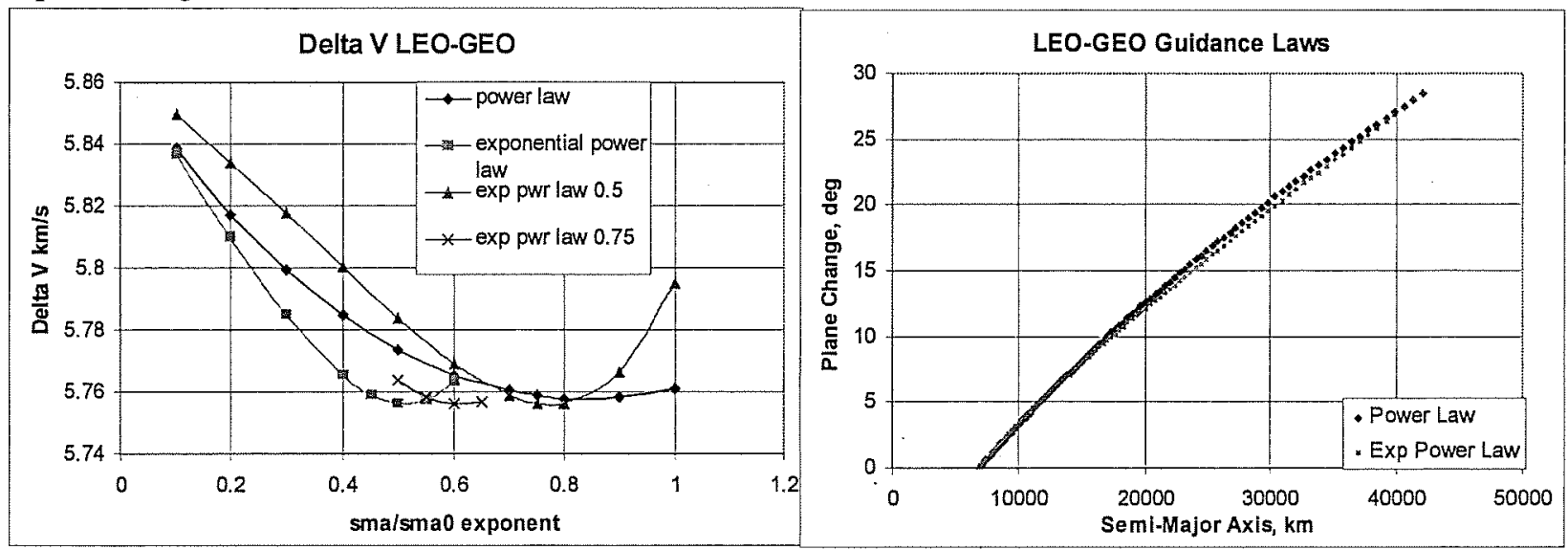

Figure 5. Results of Plane Change GN\&C Law Investigation

A low-thrust orbit becomes elliptic during raising. When one nears GEO, it is necessary to circularize. One way, not quite optimal, is to apply an apogee hold algorithm that modulates pitch when apogee reaches GEO altitude. Yaw steering continues. The equation for pitch angle $\alpha$ for apogee hold is: $\quad 2(1+e) T_{2}+2 T_{3} T_{4} / T_{2}=-$ 
$T_{3}^{2} \sin \theta /\left(T_{1} T_{2}\right) \tan \alpha$. The apogee hold pitch angle variation is such that thrusting efficiency is good only near apoapsis, so this algorithm should be used with caution.

Fig. 6 shows a detailed simulation (about 12 million integration steps) demonstrating application of the apoapsis hold algorithm at the end of the ascent (arrow). To account for both apoapsis-hold pitch and plane-change yaw, $F_{1}$ $=\left[1+\tan ^{2} \beta+\tan ^{2} \alpha\right]^{-1 / 2} ; F_{2}=F_{1} \tan \beta ; \quad F_{3}=F_{1} \tan \alpha$. The result is delta $V=5751 \mathrm{~m} / \mathrm{s}$ to end of trajectory. Loss of thrust due to solar occultations are included.

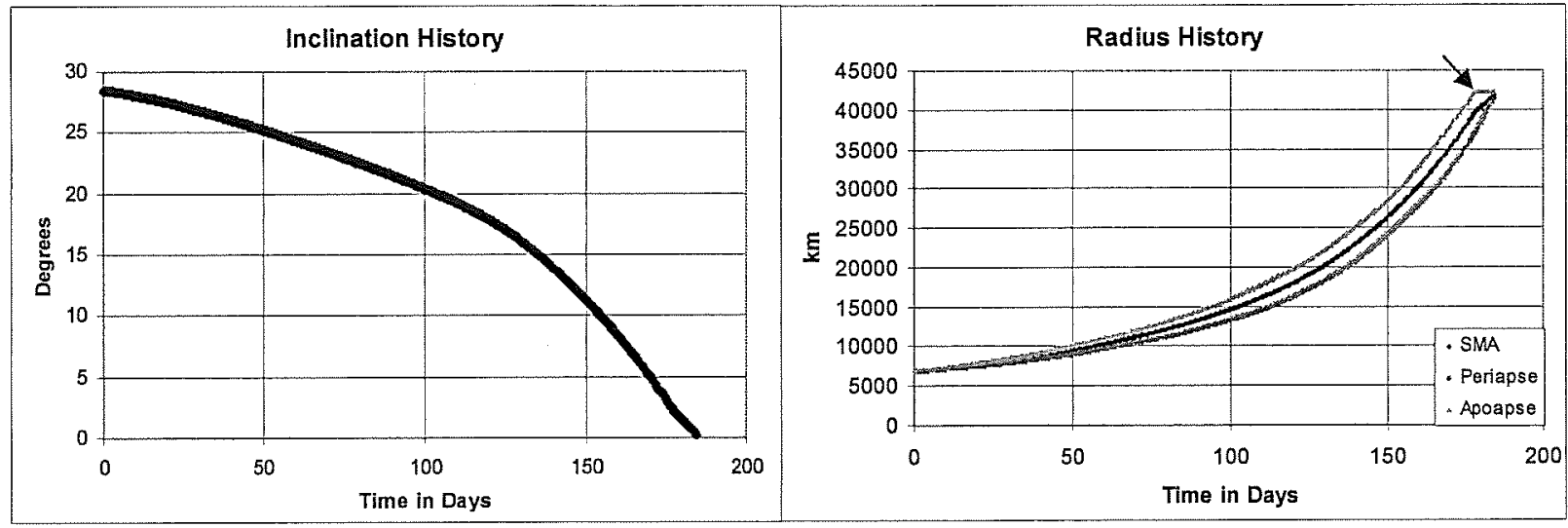

Figure 6. Plane Change Numerical Simulation Results

If one begins with an elliptic orbit, the eccentricity is enough that one should use a yaw steering law that includes eccentricity and argument of periapsis. This involves coordinate transformations. The best coordinate system in which to formulate the problem is an orbit coordinate system in which the $\mathrm{x}$ axis points through the line of nodes instead of through periapsis. The formulation used a momentum vector approach, where instantaneous plane change is given by the ratio of (1) orbital momentum vector rotation around the line of nodes due to thrust, to (2) the current orbital momentum vector. The result is:

$$
\tan \beta=\frac{\lambda_{p} \cos (\theta+\omega) \mu}{-2 \lambda_{a} a^{2} V^{2} T_{2} \cos ^{2} \gamma T_{1} T_{2}}
$$

Fig. 7 shows a representative yaw steering case for this equation, with argument of periapse zero and 30 degrees. One would normally start such an orbit raising with $\omega=0$, but secular perturbations will cause the line of apsides to advance, so it is important to include the argument of periapsis in the formula.

Fig. 8 shows results of a numerical integration with apogee hold applied when apogee reaches GEO. The orbit remains significantly elliptic until the apogee hold begins. As noted, the apogee hold algorithm is not highly efficient, as it involves large pitch angles up to $90^{\circ}$ when far from apogee. One can compute the apogee hold pitch angle and apply a fraction of it throughout the trajectory, along with yaw steering. If the right amount is applied, the eccentricity goes to

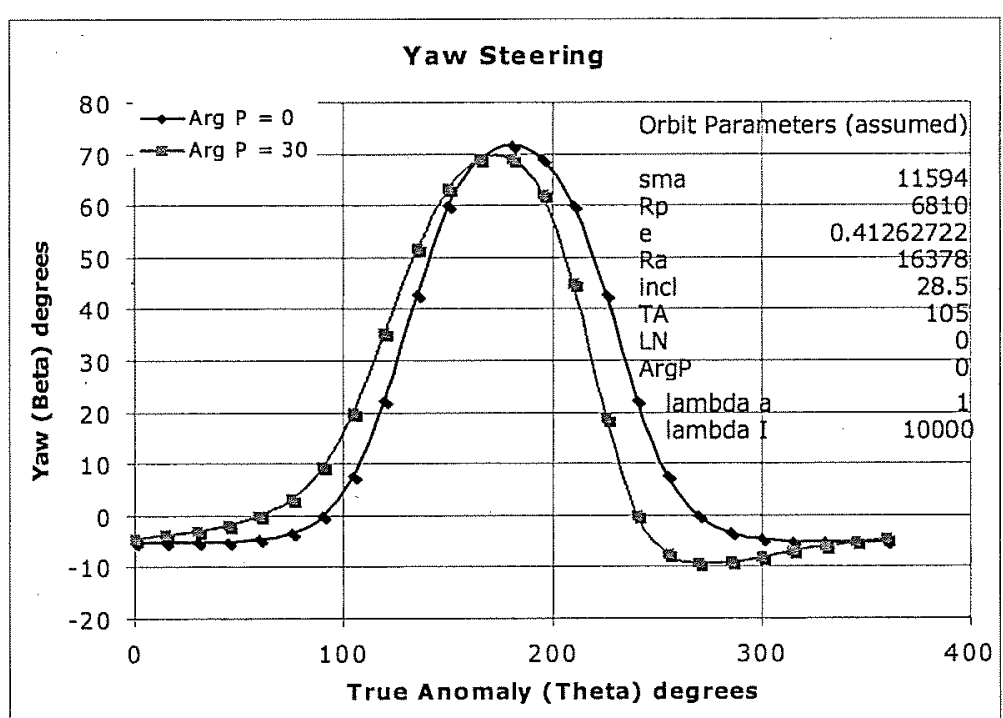

Figure 7. Typical Yaw Steering Commands Generated By Eq. 3 zero just as apogee reaches GEO. This is illustrated in Fig. 9. The lower edge of the velocity envelope for this trajectory, i.e. the velocities at apogee, is almost constant at about $3 \mathrm{~km} / \mathrm{s}$. The maximum yaw angle (near apogee) is also almost constant at about 60 degrees. Because this trajectory starts with more energy than a LEO-GEO nearcircular trajectory, it must accomplish the plane change more quickly. The integrated delta $\mathrm{V}$ is about $4200 \mathrm{~m} / \mathrm{s}$ compared with $5750 \mathrm{~m} / \mathrm{s}$ for the case starting at LEO. 


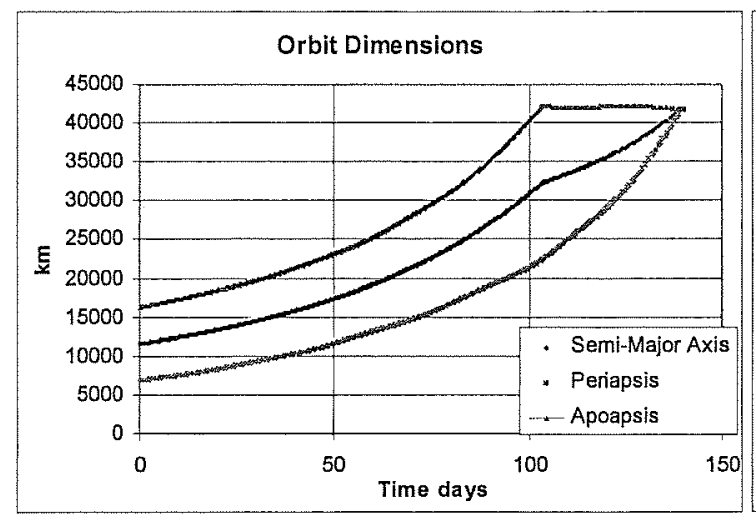

Figure 8. Elliptic Orbit Numerical Integration

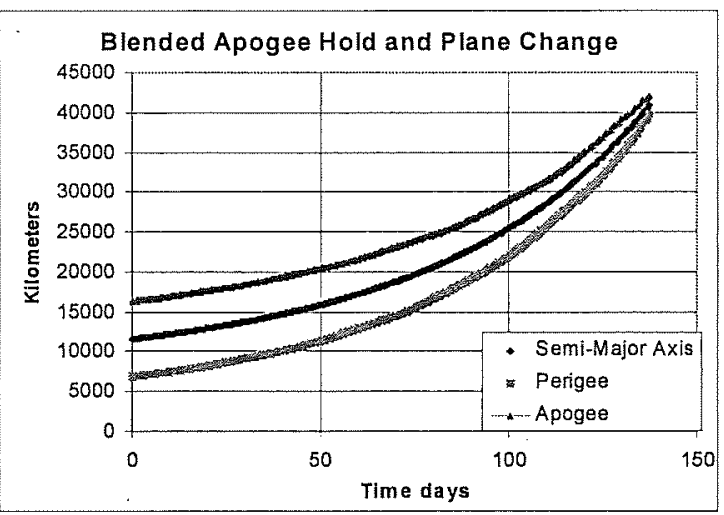

Figure 9. Elliptic Orbit, Spread Apogee Hold

\section{Selecting Specific Impulse}

The usual finding for chemical propulsion is that more specific impulse is better. That is somewhat affected by propellant density, so that in some applications the low density of hydrogen offsets (or more than offsets) its high Isp. For electric propulsion, if any limitations are placed on operating time, there is always an optimum Isp. Too low an Isp results in too much propellant consumption. Too high an Isp results in too much SEP inert mass, because the trip time limitation forces a certain thrust level, and as Isp increases, power increases causing SEP inert mass to increase. Fig. 10 illustrates this with a "simple" solar system escape trajectory not using a planet swingby. A certain minimum $\mathrm{T} / \mathrm{W}$ is required to avoid thrust from falling off faster than mass in a spiral. Delta $\mathrm{V}$ assigned was assumed to occur after Earth escape and was $50 \mathrm{~km} / \mathrm{s}$ to produce a solar system escape with significant residual velocity. Obviously, with a Jupiter swingby, solar system escape would be much easier to obtain.

This figure also illustrates existence of an optimum electric propulsion Isp. The required mass factor, $10 \mathrm{~kg} / \mathrm{kWe}$ or less for an entire SEP, is a very difficult challenge. Two optima are shown, one for payload equal SEP inert mass, which means payload increases as Isp increases because the SEP inert mass increases. The other fixes payload at $10 \%$ of the starting mass, and yields a much higher optimum Isp. Clearly,

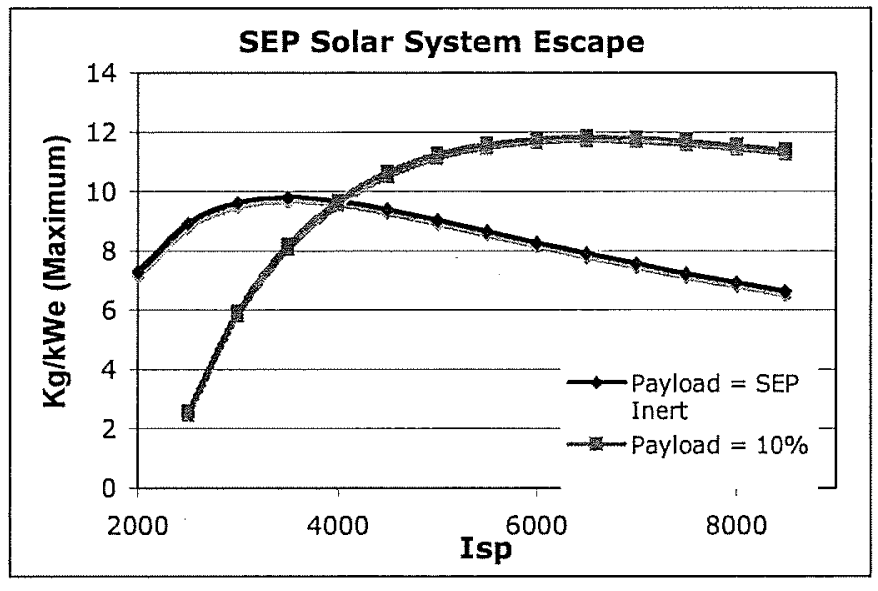

Figure 10. Optimization of SEP Isp optimization ground rules matter.

\section{Lunar Science Applications}

\section{A. Mission Profiles}

A SEP can fly from an Earth orbit to the lunar vicinity and become captured into a lunar orbit, then spiral down to a low lunar orbit, as the ESA SMART-1 did. From low lunar orbit, a lunar landing can be accomplished. Some missions need significant amounts of electric power on the lunar surface; examples are missions where the lander needs to supply power to a rover, or includes an experiment to test or demonstrate in-situ resources utilization (ISRU) processing. If significant power is included, it is tempting to use it for electric propulsion on the way to the Moon.

A mission profile was analyzed in which a combined SEP-chemical propulsion system is delivered to low Earth orbit. The chemical system propels the vehicle to an elliptic Earth orbit, to reduce trip time as well as radiation/debris exposure. The SEP takes over and delivers the vehicle to a lunar intercept. Either the SEP or the chemical system accomplishes lunar capture and reaches a low lunar orbit. The chemical system performs the lunar landing. Upon landing, the SEP solar array is re-deployed to produce power for the surface mission. This may be 
particularly applicable to resource utilization experiments, such as oxygen production. A performance augmentation could be obtained by jettisoning the SEP thrusters and power processors before the lunar landing.

A tradeoff was run to determine how much of the total delta $V$ should be delivered by the SEP, the remainder being delivered by the chemical system. The latter was assumed to use hydrogen-oxygen with Isp 450 . Delta IV Heavy was assumed for delivery to the starting elliptic orbit; payload to a typical starting orbit $250 \times 10,000 \mathrm{~km}$ is about $16,800 \mathrm{~kg}$. While Delta IV could deliver the spacecraft to TLI, we assumed the lunar landing chemical propulsion provided all chemical delta $V$ beyond the starting orbit. EP run time was held at a constant 210 days by adjusting the electric power level, except for low SEP deltaV cases. We arbitrarily assumed the power required on the surface is $25 \mathrm{kWe}$, so if $25 \mathrm{kWe}$ yielded a trip time less than 210 days we accepted the shorter trip.

Fig. 11 shows results. This is a very complex trade, and we sought only general trends. SEP Isps were 1500 and 2000. The minimum chemical delta $V$ occurs with a $1500 \mathrm{~m} / \mathrm{s}$ boost to elliptic orbit and $2100 \mathrm{~m} / \mathrm{s}$ for lunar landing. The maximum occurs with a $3000 \mathrm{~m} / \mathrm{s}$ boost (to an elliptic orbit with apogee near lunar distance), most of the lunar orbit insertion, and the landing. SEP delta $V$ ranges from a minimum of $900 \mathrm{~m} / \mathrm{s}$ to a maximum of $6010 \mathrm{~m} / \mathrm{s}$. It was assumed that portions of the mission profile on chemical propulsion would be short duration.

Maximum payload and minimum cost were found with maximum use of electric propulsion. Use of SEP imposes an obvious trip time penalty, for which we did not set a cost penalty. The sequence in which we decreased SEP delta V was first to do less lunar orbit insertion with SEP, and then to increase the starting orbit apogee, which increased the launch delta $V$ and reduced the SEP delta $V$. SEP delta $V$ to reach lunar orbit insertion from elliptic starting orbit was determined by numerical integration; a unit of launch vehicle delta $V$ was "worth" $70 \%$ more than a unit of SEP delta V. SEP delta V to spiral down to a given lunar orbit altitude was estimated as $40 \%$ more than chemical lunar orbit insertion. This difference is attributed to the difference in depth of gravity well, Earth re Moon. The result can be seen in the figure. Adding launch delta V decreases SEP power more rapidly and decreases net payload more slowly than adding chemical lunar orbit insertion delta $V$. The combination of these effects causes a reverse in the unit cost curve. A better result might be been obtained by reversing the order of delta $\mathrm{V}$ exchange, first increasing starting orbit apogee and then decreasing SEP contribution to lunar orbit insertion. When we have electric power we are not using (because surface power sets the power level) the result is disadvantageous.

Three final caveats are appropriate. The first was mentioned above, that this is a complex trade and the result is a trend analysis only. The second is that we assumed equal value for a $\mathrm{kg}$ of payload gained or launch mass reduced. In fact, there is great value in being able to use a smaller launch vehicle and much less value in saving mass if the same launch vehicle must be used. The third is that cost estimates were crude, and the result is sensitive to cost of electric propulsion relative to other costs. We used $\$ 1500 /$ watt for the aggregate of solar arrays, power processing and thrusters, and a mission cost for everything else of $\$ 350$ million. If solar electric propulsion is more costly relative to other costs than we assumed, there may be no cost benefit to this use of electric propulsion.

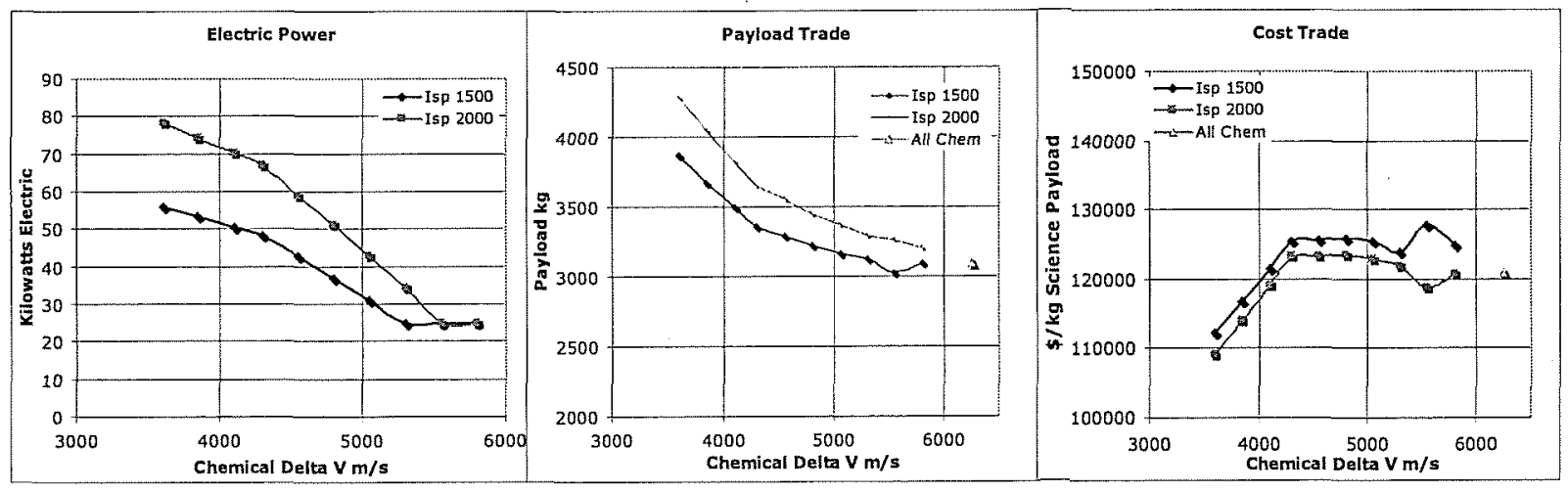

Figure 11. Results of Mixed Chemical-SEP Lunar Lander Trade

\section{B. Small Lunar SEP Study}

As part of a systems analysis for SEP applications, a design study was performed to investigate the potential for a SEP compatible with small, less expensive launch vehicles and capable of performing useful missions from low Earth orbit (LEO) to lunar orbit (LO). The launch constraints were, approximately, payload mass to LEO 550 - 600 $\mathrm{kg}$ and payload fairing $1.3 \mathrm{~m}$ diameter. Recently ${ }^{4}$, the National Research Council was reported as recommending, 
"Scientific missions to the Moon should concentrate on sampling its thin atmosphere and on examining as much of the lunar surface as possible". The small SEP is well-suited to contribute to that task.

We started with a rough allocation $200 \mathrm{~kg}$ for SEP inert mass, $200 \mathrm{~kg}$ for propellant, and $200 \mathrm{~kg}$ for payload, which was presumed to be a micro-lander. We also considered jettisoning the SEP propulsion system (it is about half the inert mass) and landing the solar array, for example as a power source for an ISRU experiment. Of course, a lunar orbit payload could also be flown.

This allocation led to an estimate that we could produce between 5 and $10 \mathrm{kWe}$ electric power, and we settled on approximately $7 \mathrm{kWe}$ for electric propulsion and a few hundred watts for spacecraft bus power. Two Hall-effect thrusters (HETs) were identified; the Aerojet BPT- $4000^{5}$ and a Glenn Research Center thruster, the NASA-103M. The former is flight-qualified for more than the propellant load for this application; the latter is developmental and is scheduled for substantial testing in 2007. These are illustrated in Figure 12. The NASA-103M is lighter and slightly higher specific impulse than the BPT-4000. It is rated at somewhat less power, $3.5 \mathrm{kWe}$, but that is as much as we need. We use 2 thrusters so that gimbal motion can provide roll control as well as pitch and yaw control.

Configuration ... The small fairing size places constraints on the configuration. It cannot be too big in diameter and must be relatively short to leave room for a payload. A basic arrangement of two propellant tanks, two solar wings and two propulsion power processing units (PPUs) was found to be relatively compact. This arrangement is shown in Figure 13. This layout fits in the fairing, leaves $1 / 2$ meter of cylinder for payload, and there is about a meter of tapered section above that. The PPUs will be able to "see space" and will almost be able to cool themselves; there's almost enough radiating area (it may in fact be enough).

Solar Array ... The small fairing diameter and need for compact packaging leads to an array configuration with small $(0.51 \times 0.75 \mathrm{~m})$ individual panels. Modern high-performance cells are relatively large, about $30 \mathrm{sq}$ $\mathrm{cm}$. A string can run either way on the panel, with length 13 cells the short dimension or 19 the longer dimension. The shorter string uses the available area slightly better with 117 cells per panel vs 114 . It also is more convenient to hook up. The arrangement is shown in Figure 14. These cells have output voltage about 2.7 , so a 13-cell string produces about $35 \mathrm{~V}$. That's pretty low voltage for $7 \mathrm{kWe}$, requiring conductors for 200 amps.

We decided to use a series-parallel hookup for the cells as shown in Figure 15. That produces about $100 \mathrm{~V}$ which interfaces better with electric propulsion PPUs, and requires less conductor mass. This requires that the array wings have a number of panels divisible by 3 ; we chose 15. There is also a half-panel inboard and outboard for symmetric folding. (Figure 16 shows one wing partly deployed.) The inboard half-panels can produce about $200 \mathrm{~W}$ at $35 \mathrm{~V}$ and are used as a backup power supply for critical vehicle functions such as communications and computing. The outboard half-panels are only structural frames for folding. Panels are deployed by single redundant-drive extensible masts in the center of the array.

Performance and Control ... The

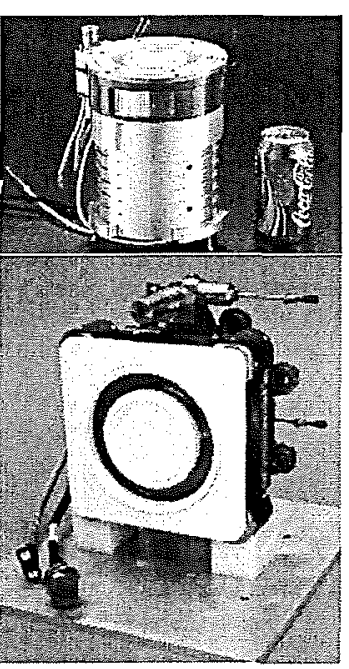

NASA-103M

- Max power: $3.5 \mathrm{~kW}$

- Thrust: $150 \mathrm{mN}$

- Specific Impulse: $1250-2750 \mathrm{sec}$

- Thruster Mass: $4.8 \mathrm{~kg}$

Estimated PPU mass $10.5 \mathrm{~kg}$

(based on $3 \mathrm{~kg} / \mathrm{kW}$ )

- Max PPU input power : $3.7 \mathrm{KW}$

( $\eta=0.95$ at full power)

Aerojet BPT-4000

- Max power: $4.5 \mathrm{~kW}$

- Thrust: $244 \mathrm{mN}$ (typ)

- Specific Impulse: 1750-2000 sec

- Thruster Mass: $12.3 \mathrm{~kg}$, includes

mounting bracket and cabling

- Estimated PPU mass $12.75 \mathrm{~kg}$

- Max PPU input power : $4.7 \mathrm{KW} \mathrm{( \eta}$ $=0.95$ at full power)

\section{Figure 12: Candidate Thrusters}

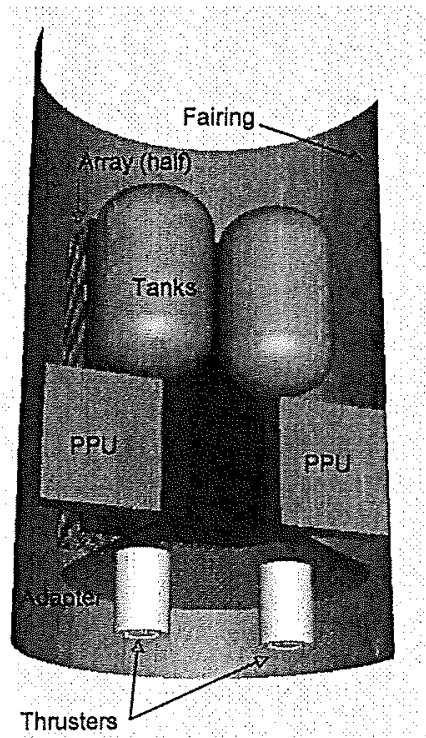

Figure 13: General Arrangement

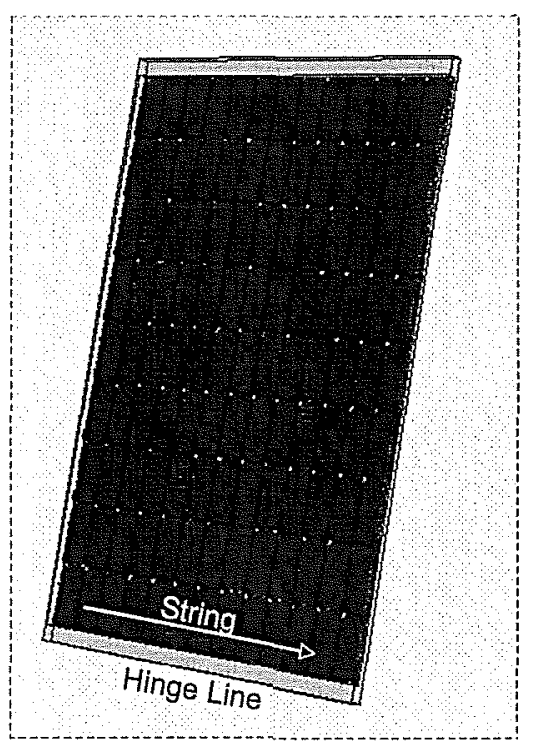

13 strings, each 9 cells long

Figure 14: Single Panel

Cell Arrangement usual method of control for a SEP vehicle 
is to roll the vehicle until the solar array long axis is perpendicular to the Sun vector and then tilt the array around this axis until it exactly faces the Sun ${ }^{6}$. The control system attempts to maintain pitch and yaw zero. Nominal thruster pointing is aft (thrust vector forward). This mode of control involves significant roll motion, hence the need for two thrusters to provide roll control. Using chemical or cold gas thrusters for this is very expensive in propellant usage, in and near low Earth orbit. (The ESA SMART-1 only had a single thruster but it started in a geosynchronous transfer orbit.)

Moments of inertia were calculated and a 6-DOF SEP flight simulation was used to assess controllability. We had some concern about the small lateral spacing of the thrusters, i.e. roll control authority. Figure 6 shows attitude motions and errors for one orbit at minimum altitude (worst case for control). The upper graphs are with no thrust during occultations and the lower are for $10 \%$ power (from a battery) during occultations. We also ran a simulation for about 45 orbits to assess performance losses due to control activity (propellant consumption versus ideal propellant consumption for the same increase in altitude); they were found to be very small, about $2 \%$. These losses will decrease with altitude. Array offpointing due to lag in attitude versus commanded attitude is slight. Power loss was calculated by a cosine rule and was negligible. The power loss would require further investigation should we decide to use a concentrator array such as the SLA.

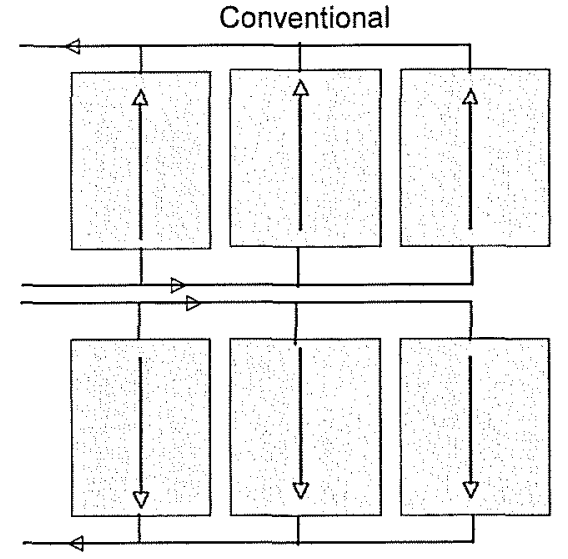
Series-parallel

Figure 15: Conventional Array Arrangement Compared with Series-Parallel

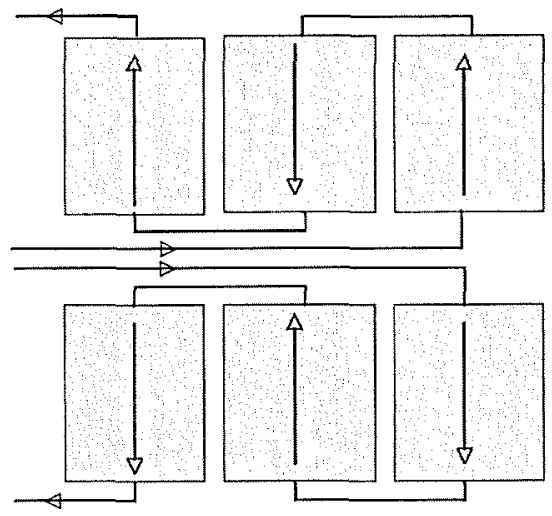

The extender is a small square section foiding truss (about $4 \times 4 \mathrm{~cm}$.) that pushes out between the array upper and lower sections While this may seem flimsy, the thrusters are only able to generate about $5 \mathrm{psi}$ stress in its members. (EP is low thrust.)

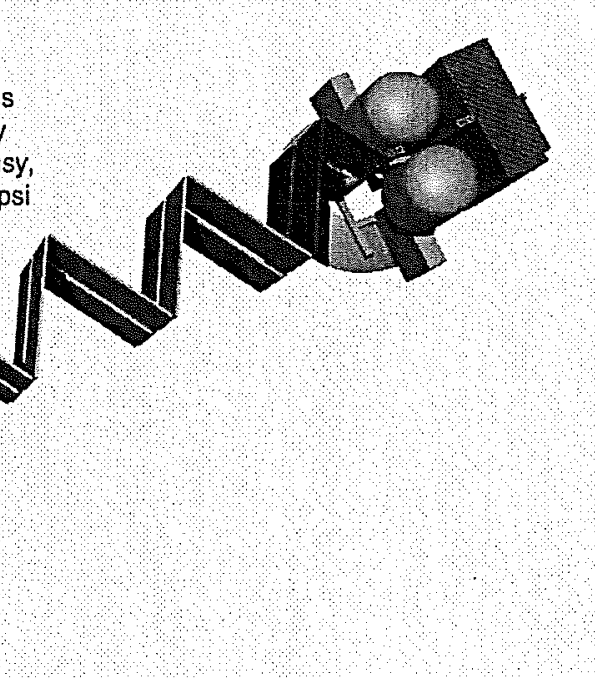

Mass Properties ...

Mass properties were

estimated from known mass of solar cells and propulsion hardware, and calculated mass for array panels and structure. The propellant tanks represent an existing design for 4500 psia helium tanks. Actual pressure rating needs to be about 2500 psia, so there is a potential for mass savings here. Adding a reasonable margin to structure and avionics adds about $30 \mathrm{~kg}$ to the dry mass. However, we think the avionics as given in Table 2 is over-estimated.

Figure 16: System With One Array Partially Extended

\begin{tabular}{|lr|}
\hline Table 2. Mass Estimate & \\
\hline Mass Summary & \\
Solar Array Installation & 40.7419875 \\
Propulsion System & 72.8 \\
Structure Frame & 29.9475016 \\
Avionics & 30.5 \\
Cold Gas ACPS & 7.83370071 \\
Dry Mass & $\mathbf{1 8 1 . 8 2 3 1 9}$ \\
Residual \& Reserve Prop & 18.8 \\
Inert Mass & $\mathbf{2 0 0 . 6 2 3 1 9}$ \\
Main Propellant & $\mathbf{1 8 8}$ \\
Payload & $\mathbf{1 8 0}$ \\
Total Mass & $\mathbf{5 6 8 . 6 2 3 1 9}$
\end{tabular}



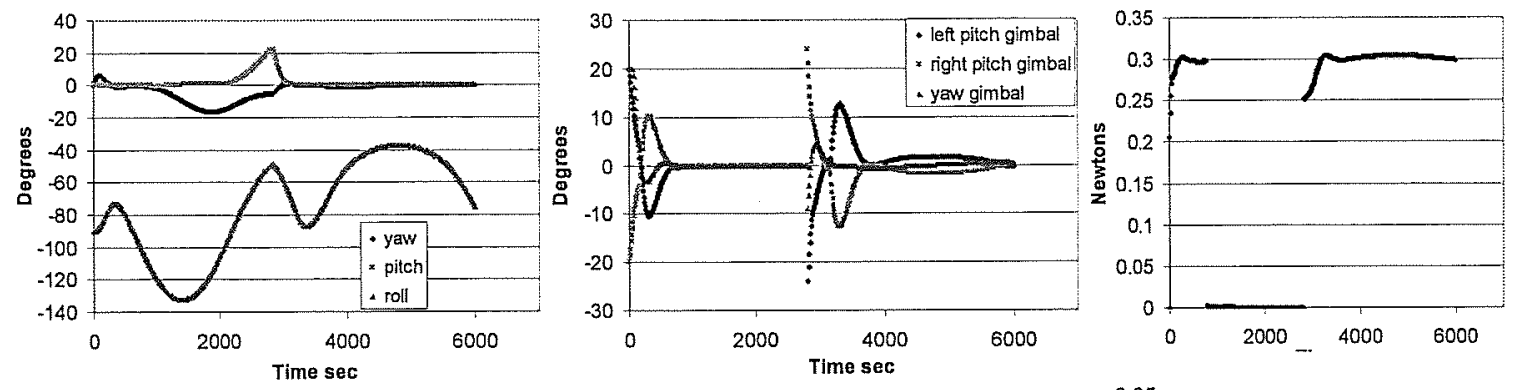

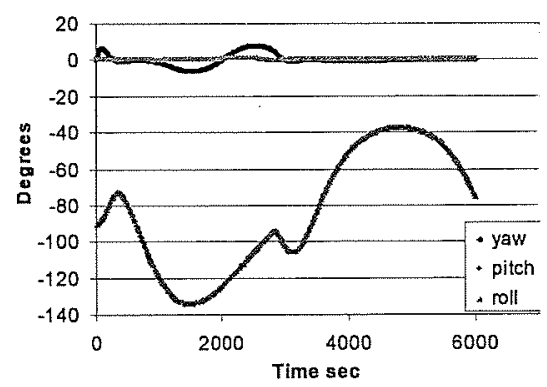

(a) Vehicle Attitude

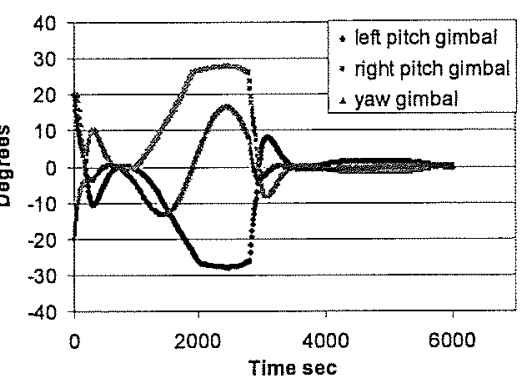

(b) Gimbal Motion

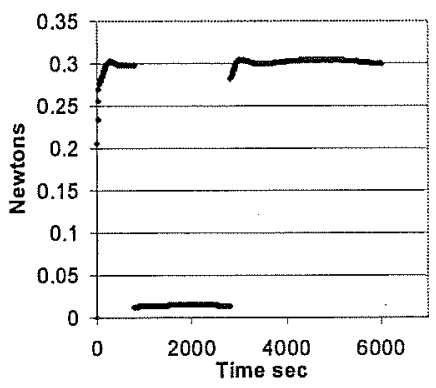

(c) Thrust

Figure 17: Results of 6-DOF Flight Simulation for One Orbit: top - no battery; bottom - 5\% battery

\section{Planetary Science Applications}

In this section, we describe a near-Earth SEP trajectory option that may improve performance for planetary science missions.

Deep Space I was something of a small template for SEP planetary missions. It had a single NSTAR thruster rated at about $2.5 \mathrm{kWe}$, and visited the comet Borrelly. Numerous studies of larger SEP spacecraft for outer planet missions have identified payoffs ${ }^{7}$. These concepts have typically been rated at about 25 kwe and used 3 to $5 \mathrm{NEXT}^{7}$ thrusters rated at about $6 \mathrm{kWe}$.

Outer planet trajectories from these studies have similar characteristics. The SEP is launched to a C3 about 10 to 15. It attains a trajectory with aphelion somewhere beyond Mars, and adjusts its perihelion to about the radius of Venus. A coast period ensues, followed by a thrusting period through the perihelion arc, usually including a Venus gravity assist (VGA). (This type of trajectory works without VGA but works better with it.) Thrusting continues to about the distance of Mars, by which time the SEP has achieved enough energy to reach whichever outer planet is selected. The SEP then ceases propulsion and can be jettisoned. The scientific spacecraft bus carries aerocapture and/or chemical propulsion, or possibly radioisotope electric propulsion for maneuvers in the outer solar system since the SEP is too far from the Sun to provide useful thrust.

These trajectories, including the launch condition, are optimized by SEPTOP or a similar code. Because launch vehicle high-thrust propulsion has high leverage deep in Earth's gravity well, the optimizer increases C3 until the SEP leverage due to its high Isp exceeds the high-thrust leverage. Fig 18 graphs the relative leverage of high thrust and SEP propulsion versus $\mathrm{C} 3$. If one starts the optimization at C3 0 or greater, the result will be as noted, C3 $10-$ 15. However, there is a region at $\mathrm{C} 3<0$ where the SEP also has good leverage.

This was investigated with a simple spread-sheet analysis to test the hypothesis that a SEP start at $\mathrm{C} 3<0$ might have payoff. At first, a two-stage SEP was postulated, starting in LEO with the booster delivering to $\mathrm{C} 3$ about zero (delta $\mathrm{V}$ about $7 \mathrm{~km} / \mathrm{s}$ ) and a second stage delivering the delta $V$ to go from $\mathrm{C} 3=0$ to outer planet injection; this delta $\mathrm{V}$ is about 18 to $20 \mathrm{~km} / \mathrm{s}$. The SEP booster could be re-usable.

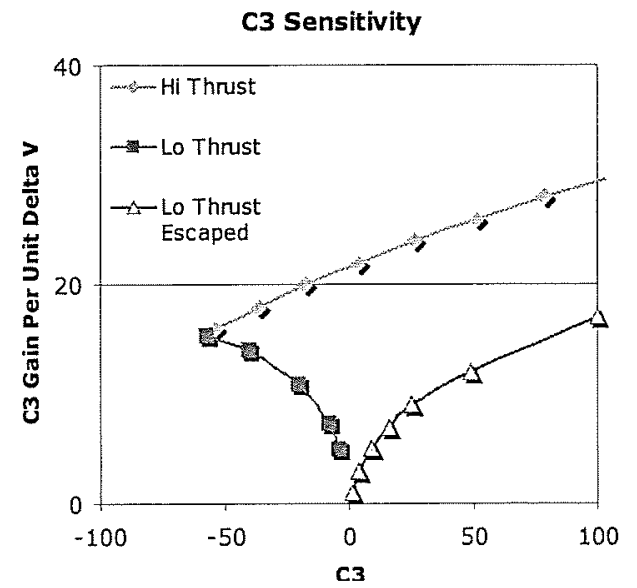

Figure 18. SEP Leverage vs. High-Thrust 
The Excel solver was used to do a simple constrained optimization (this was not a trajectory analysis; representative ideal delta Vs were used). It was found that the booster and mission stages had similar power level, and that Isp optimized about 2200 for the booster and 3000 for the mission stage. It was then realized that one could use Hall thrusters with two-step voltage supply to do the Isp switch and eliminate the booster, thus eliminating one set of solar arrays, power processors and thrusters. Crude cost estimating indicated about $25 \%$ to $30 \%$ cost and mass savings compared to the usual solution with launch to $\mathrm{C} 3 \sim 10$ to 15 . The power level was $\sim 50 \mathrm{kWe}$. This trajectory concept was checked with a Dawn mission-like trajectory using SEPTOP. Net delivered mass increased by about $50 \%$ at the expense of 6 months more trip time. This trajectory type might offer opportunity for a smaller launch vehicle in some cases. It would also be useful where the launch vehicle does not have a high-performance upper stage.

\section{Conclusions and Recommendations}

SEP systems offer much promise for improving mission performance and decreasing costs. A wide range of mission applications show benefits. There is a progression in power level from current missions at about $5 \mathrm{kWe}$, through lunar and planetary science applications at $20-80 \mathrm{kWe}$ and orbit raising for communications satellites at 30 $-100 \mathrm{kWe}$, all the way to support roles for human exploration and exploitation missions (not discussed in this paper), at hundreds of $\mathrm{kWe}$ to a megawatt or more. Some of these applications admit to incremental mission architecture improvements, with accompanying reductions in cost. Solar array performance continues to increase, and concentrator systems promise to improve radiation resistance for orbit raising, so that the requisite re-use of orbit raising SEP "tugs" (needed for cost reduction) can be achieved.

Potential mission applications are numerous, including space science, space exploration, and present and future commercial uses of space. A coordinated technology advancement and mission technology infusion effort would benefit all users. Commercial customers are likely to have less risk exposure in terms of lost time, and may be first users of some advancements, as they have in the past. However, commercial customers are unlikely to lead the way in technology development.

\section{Acknowledgement}

The work described in this paper was funded by the In-Space Propulsion Technology Program, managed by NASA's Science Mission Directorate in Washington, D.C., and implemented by the In-Space Propulsion Technology Project at Marshall Space Flight Center in Huntsville, Alabama. The program objective is to develop in-space propulsion technologies that can enable or benefit near and mid-term NASA space science missions by significantly reducing cost, mass or travel times.

\section{References}

1. Woodcock, Gordon R. and Dankanich, John, "Application of Solar-Electric Propulsion to Robotic and Human Missions in Near-Earth Space", AIAA Joint Propulsion Conference, 2006

2. Isakowitz, S. J., Hopkins, J. B., and Hopkins, J. P., International Reference Guide to Space Launch Systems, Fourth Edition, AIAA, 2004

3. Low-Thrust Technology Interchange Meeting, held at MSFC March 28 \& 29, 2006

4. Fox, Maggie, "Report lays out science map for return to the Moon", Reuters news report, Sept 19, 2006

5. De Grys, K.; Welander, Ben; Dimicco, John; Wenzel, Scott; and Kay, Bob; "4.5 kW Hall Thruster System Qualification Status". AIAA-2005-3682, AIAA Joint Propulsion Conference, 2005

6. Woodcock, G. "Controllability of Large SEP for Earth Orbit Raising", AIAA Joint Propulsion Conference, 2004

7. Cupples, M., Coverstone, V. and Woo, B., "Application of Solar Electric Propulsion to a Comet Surface Sample Return Mission", AIAAJASME Joint Propulsion Conference, 12 July 2004, Ft. Lauderdale FL 